KEK-TH-1261

\title{
Charged Black Holes in a Rotating Gross-Perry-Sorkin Monopole Background
}

\author{
Shinya Tomizawa ${ }^{1}$ and Akihiro Ishibashi ${ }^{1,2}$ \\ ${ }^{1}$ Cosmophysics Group, \\ Institute of Particle and Nuclear Studies, \\ KEK, Tsukuba, Ibaraki, 305-0801, Japan \\ and \\ ${ }^{2}$ Perimeter Institute for Theoretical Physics, \\ Waterloo, ON N2L 2Y5, Canada
}

(Dated: October 29, 2018)

\begin{abstract}
We present a new class of stationary charged black hole solutions to five-dimensional EinsteinMaxwell-Chern-Simons theories. We construct the solutions by utilizing so called the squashing transformation. At infinity, our solutions behave as a four-dimensional flat spacetime plus a 'circle' and hence describe a Kaluza-Klein black hole. More precisely, our solutions can be viewed as a charged rotating black hole in a rotating Gross-Perry-Sorkin monopole background with the black hole rotation induced from the background rotation.
\end{abstract}

PACS numbers: 04.50.+h 04.70.Bw 


\section{INTRODUCTION}

In recent years, higher dimensional black holes have become one of the major subjects in fundamental physics $\lfloor$ 1, 2, , 3, 4, 5, 6, 7, 8, 9, 10, 11, 12, 13, 14, 15, 16, 17, 18, 19]. Although recent ideas of braneworld and TeV gravity [20, 21, 22] have opened up the possibility of 'large' extra-dimensions, still important and widely believed especially in the context of string theories is the spacetime picture that our macroscopically large four-dimensional spacetime is realized from a higher dimensional spacetime by some mechanism of compactifying (and stabilizing) extra-dimensions within the size of the fundamental scale of gravity. In this context, it is of great interest to consider Kaluza-Klein black holes, which are essentially higher dimensional near the event horizon but look like four-dimensional with compactified small extra-dimensions, at large distances.

The purpose of this paper is to provide a new class of Kaluza-Klein black hole solutions, which are constructed by applying so called the squashing transformation (described below) to a known starting-point solution in five-dimensional theories. Specifically, we consider the Reissner-Nordstöm-Gödel black hole solution of Herdeiro [23] in five-dimensional EinsteinMaxwell-Chern-Simons theories, as our starting-point geometry. We "squash" it, thereby obtaining a new metric specified by 4 parameters. We then choose the parameters so that the resultant spacetime possesses two Killing horizons. As a result, our new solutions have the black hole event horizon whose cross-section geometry is of squashed $\mathrm{S}^{3}$, as well as an inner horizon, and are asymptotically locally flat with the structure of a twisted $\mathrm{S}^{1}$ bundle over a four-dimensional Minkowski spacetime. It is remarkable to note that although our starting-point geometry [23] admits closed timelike curves as a consequence of Gödel type rotation, our resultant new Kaluza-Klein solution itself exhibits no causal pathology outside the event horizon, thanks to the squashing transformation and appropriate choice of the parameters.

Our Kaluza-Klein black holes obtained in the way described above can also be viewed as a charged rotating black hole in the rotating Gross-Perry-Sorkin (GPS) monopole background [24, 25], which itself is obtained via the squashing transformation from the fivedimensional Gödel universe in Einstein-Maxwell-Chern-Simons theories. Therefore, in order 
to understand basic properties of our Kaluza-Klein solutions, it would be illuminating to discuss first the geometry and basic features of the rotating GPS background solutions. We shall do so in the next section. But before proceeding subsequent sections and presenting our solutions, we would like to review briefly some related work.

The first example of such Kaluza-Klein black holes was given as a vacuum solution to the five-dimensional Einstein equation by Dobiasch and Maison [26]. It was then shown by Gibbons and Wiltshire [27] that the solution of Dobiasch and Maison asymptotes to a twisted $\mathrm{S}^{1}$ bundle over the four-dimensional spacetime with a single rotation with respect to the extra fifth-dimension. (See e.g., Rasheed [28] for further generalization of the Dobiasch and Maison solution [26].)

A recent key work along this line is that of Ishihara and Matsuno [29], who have found static charged Kaluza-Klein black hole solutions in the five-dimensional Einstein-Maxwell theory by using, for the first time, the squashing technique. Namely, they view the base manifold $\mathrm{S}^{3}$ of a five-dimensional Reissner-Nordstöm black hole as a fiber bundle over $\mathrm{S}^{2}$ with fiber $S^{1}$ and then consider a deformation that changes the ratio of the radius of $S^{2}$ to that of $\mathrm{S}^{1}$ fiber. Subsequently the Ishihara-Matsuno solution was generalized to many different cases. For example, a static multi Kaluza-Klein black hole solution [30] was constructed immediately from the Ishihara-Matsuno solution. The squashing transformation of IshiharaMatsuno [29], which we will also employ in this paper, has been then recognized as a type of powerful solution generating technique. In fact, it was demonstrated by Wang [31] that the five-dimensional Kaluza-Klein black hole of Dobiasch and Maison can be reproduced by squashing a five-dimensional Myers-Perry black hole with two equal angular momenta.

The application of the squashing transformation to non-asymptotically flat Kerr-Gödel black hole solutions [32] was considered in [25]. In [33], the squashing transformation was applied to the Cvetic et al's charged rotating black hole solution [13] with two equal angular momenta. These solutions [25, 33] correspond to a generalization of the Ishihara-Matsuno solution to the rotating black holes in Einstein-Maxwell-Chern-Simons theories. A similar type of Kaluza-Klein black holes was considered in the context of supersymmetric theories by Gaiotto et al [34] and Elvang et al [35]. Kaluza-Klein black holes which asymptote to 
the direct product of the four-dimensional Minkowski space-time and $\mathrm{S}^{1}$ were discussed by [36, 37, 38]. Further generalizations of Kaluza-Klein solutions of this type and their basic properties have been studied by many authors [39, 40, 41, 42, 43, 44, 45, 46, 47, 48].

The rest of this paper is organized as follows. In the next section, as mentioned above, we will review the five dimensional Gödel universe and the rotating GPS monopole based on the results of the paper [25]. Then, in section III, we present our new stationary charged Kaluza-Klein black hole solutions in the rotating GPS monopole background. In section IV, we examine our solutions under several special choices of the parameters and study their basic properties. Section $\nabla$ is devoted to summary and discussions. In Appendix we show that our background, rotating GPS monopole geometry is a supersymmetric solution.

\section{ROTATING GROSS-PERRY-SORKIN MONOPOLE}

We shall briefly review the results of [25] concerning the rotating Gross-Perry-Sorkin (GPS) monopole, which is one of the simplest solutions in five-dimensional Einstein-MaxwellChern-Simons theories. We begin with the five-dimensional Gödel universe since the rotating GPS monopole solutions are obtained by applying the squashing transformation to the fivedimensional supersymmetric Gödel universe. We then discuss some basic properties of the rotating GPS monopole, in particular, its asymptotic structure and the existence of an ergoregion.

\section{A. Five-dimensional Gödel universe}

Consider the five-dimensional Einstein-Maxwell theory with a Chern-Simons term, whose action is given by

$$
S=\frac{1}{16 \pi G_{5}} \int d^{5} x\left[\sqrt{-g}\left(R-F_{\mu \nu} F^{\mu \nu}\right)+\frac{2}{3 \sqrt{3}} \epsilon^{\mu \nu \rho \sigma \lambda} A_{\mu} F_{\nu \rho} F_{\sigma \lambda}\right],
$$

where $R$ is the five dimensional scalar curvature, $F=d A$ is the two-form of the fivedimensional gauge field associated with the gauge potential one-form $A$, and $G_{5}$ is the 
five-dimensional Newton constant. Varying the action (11), we derive the Einstein equation

$$
R_{\mu \nu}-\frac{1}{2} R g_{\mu \nu}=2\left(F_{\mu \lambda} F_{\nu}^{\lambda}-\frac{1}{4} g_{\mu \nu} F_{\rho \sigma} F^{\rho \sigma}\right)
$$

and the Maxwell equation

$$
F_{; \nu}^{\mu \nu}+\frac{1}{2 \sqrt{3}}(\sqrt{-g})^{-1} \epsilon^{\mu \nu \rho \sigma \lambda} F_{\nu \rho} F_{\sigma \lambda}=0 .
$$

Then, as a solution, we obtain the following metric and the gauge potential one-form, respectively,

$$
d s^{2}=-\left(d t+j r^{2} \sigma_{3}\right)^{2}+d r^{2}+\frac{r^{2}}{4}\left(\sigma_{1}^{2}+\sigma_{2}^{2}+\sigma_{3}^{2}\right)
$$

and

$$
A=\frac{\sqrt{3}}{2} j r^{2} \sigma_{3}
$$

where the one-forms $\sigma_{i}(i=1,2,3)$ are given by

$$
\begin{aligned}
\sigma_{1} & =\cos \psi d \theta+\sin \psi \sin \theta d \phi, \\
\sigma_{2} & =-\sin \psi d \theta+\cos \psi \sin \theta d \phi, \\
\sigma_{3} & =d \psi+\cos \theta d \phi,
\end{aligned}
$$

with the coordinates $(r, \theta, \phi, \psi)$ having the ranges $0<r<\infty, 0 \leq \theta<\pi, 0 \leq \phi<2 \pi$, $0 \leq \psi<4 \pi$. The parameter, $j$, is called the Gödel parameter.

The norm of the Killing vector $\psi^{\mu}:=(\partial / \partial \psi)^{\mu}$ becomes negative in the region of $r>1 /(2|j|)$ with the signature remaining Lorentzian, and therefore this solution admits closed timelike curves (CTCs), as the four-dimensional Gödel universe does. The metric, eq. (4), is completely homogeneous as a five-dimensional spacetime, just like the fourdimensional Gödel universe is so in the four-dimensional sense. While the four-dimensional Gödel universe is a spacetime filled with a pressureless perfect fluid balanced with a negative cosmological constant, the five-dimensional Gödel universe given above, eqs. (44) and (15), is filled with a pressureless dust only, as immediately seen from its energy-momentum tensor

$$
T^{\mu \nu}=\frac{1}{4 \pi}\left(F^{\mu \nu}-\frac{1}{4} g^{\mu \nu} F^{2}\right)=\frac{3 j^{2}}{\pi}(\partial / \partial t)^{\mu}(\partial / \partial t)^{\nu},
$$

where it should be noted that the energy density itself is constant. 


\section{B. A rotating GPS monopole}

The rotating GPS monopole solution is given by the following metric and the gauge potential one-form:

$$
\begin{aligned}
d s^{2} & =-\left(d t+j r^{2} \sigma_{3}\right)^{2}+k(r)^{2} d r^{2}+\frac{r^{2}}{4}\left[k(r)\left(\sigma_{1}^{2}+\sigma_{2}^{2}\right)+\sigma_{3}^{2}\right], \\
A & =\frac{\sqrt{3}}{2} j r^{2} \sigma_{3},
\end{aligned}
$$

where $k(r)$ - called the squashing function - is given by

$$
k(r)=\frac{r_{\infty}^{4}}{\left(r_{\infty}^{2}-r^{2}\right)^{2}} .
$$

It is immediate to see that the metric of the Gödel universe, eq. (4), is transformed as

$d r \rightarrow k(r) d r, \sigma_{1} \rightarrow \sqrt{k(r)} \sigma_{1}$ and $\sigma_{2} \rightarrow \sqrt{k(r)} \sigma_{2}$. By this transformation, the metric of the unit round $\mathrm{S}^{3}$ is deformed to the metric of a squashed $\mathrm{S}^{3}$ for which the radius of $\mathrm{S}^{2}$ is no longer equal to the radius of $\mathrm{S}^{1}$. (See the part of the metric in the square bracket of eq. (10).) For this reason, this is called the squashing transformation. The range of the coordinate $r$ is $0<r<r_{\infty}$. The point $r=r_{\infty}$ turns out to correspond to spatial infinity.

Now let us introduce the following new radial coordinate

$$
\rho=\frac{r_{\infty} r^{2}}{2\left(r_{\infty}^{2}-r^{2}\right)}
$$

so that $r \rightarrow r_{\infty}$ corresponds to $\rho \rightarrow \infty$. Then, the metric and the gauge potential one-form can be rewritten, respectively, as

$$
\begin{gathered}
d s^{2}=-\left[d t+4 j \rho_{0}^{2}\left(1+\frac{\rho_{0}}{\rho}\right)^{-1} \sigma_{3}\right]^{2} \\
+\left(1+\frac{\rho_{0}}{\rho}\right)\left[d \rho^{2}+\rho^{2}\left(\sigma_{1}^{2}+\sigma_{2}^{2}\right)\right]+\rho_{0}^{2}\left(1+\frac{\rho_{0}}{\rho}\right)^{-1} \sigma_{3}^{2}, \\
A=2 \sqrt{3} \rho_{0}^{2} j\left(1+\frac{\rho_{0}}{\rho}\right)^{-1} \sigma_{3},
\end{gathered}
$$

where we have also introduced the constant $\rho_{0}=r_{\infty} / 2$. 
In this spacetime, depending on the choice of parameter range, we suffer from causality violation due to the existence of closed timelike curves (CTCs). (Recall that $\sigma_{3}$ in the first term of the above metric includes the periodic coordinate $\psi$.) To cure this, we hereafter restrict the range of the parameters $\left(j, \rho_{0}\right)$ as

$$
j^{2}<\frac{1}{16 \rho_{0}^{2}}, \quad \rho_{0}>0 .
$$

When the Gödel parameter is $j=0$, the metric, eq. (14), coincides with the static GPS monopole solution given originally in Ref. [24]. In this case it is immediate to see that the point of $\rho=0$ is a fixed point of the Killing vector field, $\psi^{\mu}=(\partial / \partial \psi)^{\mu}$, and the metric is analytic there, and thus the metric corresponds to a Kaluza-Klein monopole [24]. A fixed point of some Killing field like this is often called a nut. This is also the case even when $j \neq 0$.

Further, introduce new coordinates defined by

$$
\bar{t}=\frac{t}{C}, \quad \bar{\psi}=\psi-\frac{D}{C} t,
$$

where the constants $C$ and $D$ are

$$
C=\sqrt{1-16 j^{2} \rho_{0}^{2}}, \quad D=\frac{4 j}{\sqrt{1-16 j^{2} \rho_{0}^{2}}},
$$

which make sense under the condition, eq. (16). For $\rho \rightarrow \infty$, the metric behaves as

$$
d s^{2} \simeq-d \bar{t}^{2}+d \rho^{2}+\rho^{2}\left(\sigma_{1}^{2}+\sigma_{2}^{2}\right)+\rho_{0}^{2}\left[1-16 j^{2} \rho_{0}^{2}\right] \sigma_{3}^{2} .
$$

It is now clear that the metric is asymptotically locally flat and has the structure of a twisted $\mathrm{S}^{1}$ bundle over the four-dimensional Minkowski space-time. It is also clear that the presence of non-vanishing parameter $j$ means that although the spacetime, eq. (14), has no black hole, it is rotating along the direction of the extra-dimension specified by $\psi^{\mu}$. We emphasize here again that CTCs which exist in the Gödel universe, eq. (4), now cease to exist as a result of the squashing transformation and the choice of the parameter range, eq. (16).

Remarkably, this rotating GPS monopole solution possesses an ergoregion, despite the fact that there is no black hole event horizon in this spacetime. This can be seen as follows. 
The $\overline{t t}$-component of the metric in the rest frame takes the following form near infinity,

$$
g_{\overline{t t}}=-\left[\left(C+\frac{1-C^{2}}{C}\left(1+\frac{\rho_{0}}{\rho}\right)^{-1}\right)^{2}-\frac{1-C^{2}}{C^{2}}\left(1+\frac{\rho_{0}}{\rho}\right)^{-1}\right]
$$

As shown in FIG.1, in the case of $\sqrt{3} / 8<|j| \rho_{0}<1 / 4, g_{\overline{t t}}$ becomes positive in the region of $\gamma_{-}<\rho<\gamma_{+}$, where

$$
\gamma_{ \pm}:=\rho_{0} \frac{1-3 C^{2} \pm\left(1-C^{2}\right) \sqrt{1-4 C^{2}}}{2 C^{2}}
$$

and therefore there exists an ergoregion in that region, although there is no black hole horizon in the space-time. In a neighborhood of the nut, the ergoregion vanishes.

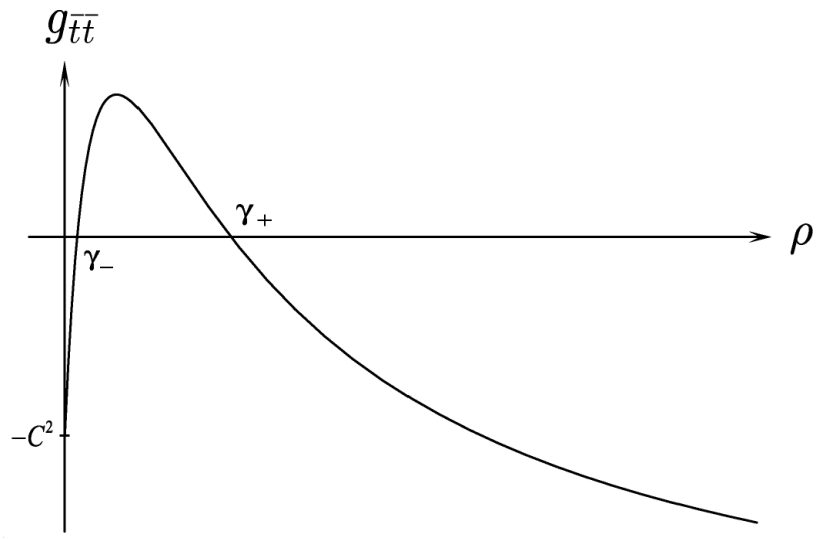

FIG. 1: The typical behavior of $g_{\bar{t} \bar{t}}$ in the case of $\sqrt{3} / 8<|j| \rho_{0}<1 / 4$. There exists an ergoregion in the region such that $g_{\overline{t t}}>0$.

The energy-momentum tensor for the rotating GPS monopole solution, eqs. (10) and (11), is calculated as

$$
T^{\mu \nu}=\frac{3 j^{2}}{\pi k(r)^{2}}(\partial / \partial t)^{\mu}(\partial / \partial t)^{\nu}
$$

Hence the rotating GPS monopole spacetime is filled with the pressureless perfect fluid with the same form of the energy-momentum tensor for the five-dimensional Gödel universe. However, the energy-density is no longer constant, in contrast to the five-dimensional Gödel universe, and falls off at large distances as the squashing function behaves $k\left(r_{\infty}\right) \rightarrow \infty(r \rightarrow$ $\left.r_{\infty}\right)$. Thus, the rotating GPS monopole solution asymptotically approaches a locally flat, 
vacuum spacetime. Furthermore, it can be shown by following Gauntlett et al. [49] that the rotating GPS monopole is a supersymmetric solution on the self-dual Euclidean Taub-NUT base space (see Appendix).

\section{BLACK HOLES IN A ROTATING GPS MONOPOLE}

\section{A. Metric and gauge potential}

Now we present our new Kaluza-Klein black hole solutions to the equations (2)-(3) . The metric and gauge potential are given, respectively, by

$$
d s^{2}=-f(r) d t^{2}-2 g(r) \sigma_{3} d t+h(r) \sigma_{3}^{2}+\frac{k(r)^{2} d r^{2}}{V(r)}+\frac{r^{2}}{4}\left[k(r)\left(\sigma_{1}^{2}+\sigma_{2}^{2}\right)+\sigma_{3}^{2}\right]
$$

and

$$
A=\frac{\sqrt{3}}{2}\left[\frac{q}{r^{2}} d t+\left(j r^{2}+2 j q\right) \sigma_{3}\right]
$$

where the metric functions, $f(r), g(r), h(r)$, and $V(r)$ are

$$
\begin{aligned}
& f(r)=1-\frac{2 m}{r^{2}}+\frac{q^{2}}{r^{4}} \\
& g(r)=j r^{2}+3 j q \\
& h(r)=-j^{2} r^{2}\left(r^{2}+2 m+6 q\right) \\
& V(r)=1+\frac{-2 m+16 j^{2}(m+q)(m+2 q)}{r^{2}}+\frac{q^{2}\left(1-8 j^{2}(m+3 q)\right)}{r^{4}}
\end{aligned}
$$

and the squashing function $k(r)$ is

$$
k(r)=\frac{V\left(r_{\infty}\right) r_{\infty}^{4}}{\left(r^{2}-r_{\infty}^{2}\right)^{2}}
$$

The one-forms $\sigma_{i}(i=1,2,3)$ are given by eqs. (8) . The range of the "spatial" coordinates $(r, \psi, \phi, \theta)$ is $0<r<r_{\infty}, 0 \leq \psi<4 \pi, 0 \leq \phi<2 \pi$, and $0 \leq \theta<\pi$. The spacetime has the timelike Killing vector field, $\xi^{\mu}=(\partial / \partial t)^{\mu}$, and two Killing vector fields, $\psi^{\mu}$ and $\phi^{\mu}=(\partial / \partial \phi)^{\mu}$ with closed orbits.

The metric above can be viewed, on one hand, as a squashed Reissner-Nordström-Gödel black hole since the metric recovers that of the Reissner-Nordström-Gödel black hole solution [23] when one takes the limit $r_{\infty} \rightarrow \infty$ with keeping the other parameters finite, i.e., 
$k(r) \rightarrow 1$. (Note that $\psi^{\mu}$ can be timelike in this limit and CTCs appear.) On the other hand, if one takes the limit $m \rightarrow 0$ and $q \rightarrow 0$, then the metric recovers the rotating GPS monopole spacetime discussed in the previous sections and hence can be viewed as a charged black hole on the rotating GPS monopole background.

In what follows the 4 parameters $\left(j, m, q, r_{\infty}\right)$ that specify the above solutions are assumed to satisfy the following inequalities,

$$
\begin{aligned}
& r_{\infty}^{2}>m-8 j^{2}(m+q)(m+2 q)>0, \\
& 1-8 j^{2}(m+3 q)>0, \\
& {\left[m-q-8 j^{2}(m+q)^{2}\right]\left[m+q-8 j^{2}(m+2 q)^{2}\right]>0,} \\
& r_{\infty}^{4}+2\left[-m+8 j^{2}(m+q)(m+2 q)\right] r_{\infty}^{2}+q^{2}\left(1-8 j^{2}(m+3 q)\right)>0, \\
& r_{\infty}^{2}<\frac{1}{4 j^{2}}-(2 m+6 q) .
\end{aligned}
$$

As we will see below, these inequalities are the necessary and sufficient conditions for the spacetime, eq. (23) , to admit two Killing horizons and no CTCs outside the horizon. The parameter region is shown in FIG.2 and 3, in which the parameters $\left(m, q, r_{\infty}\right)$ are normalized by $j$ as $M=j^{2} m, Q=j^{2} q, R_{\infty}^{2}=j^{2} r_{\infty}^{2}$, with the parameter $r_{\infty}$ being fixed. $C_{i}(i=1, \cdots, 6)$ denote the following curves in the $(Q, M)$-planes:

$$
\begin{aligned}
& (C 1) M-Q-8(M+Q)^{2}=0, \\
& (C 2) M-8(M+Q)(M+2 Q)=0, \\
& (C 3) M-8(M+Q)(M+2 Q)=R_{\infty}^{2}, \\
& (C 4) M+Q-8(M+2 Q)^{2}=0, \\
& (C 5) R_{\infty}^{4}+2(-M+8(M+Q)(M+2 Q)) R_{\infty}^{2}+Q^{2}(1-8(M+3 Q))=0, \\
& (C 6) R_{\infty}^{2}=\frac{1}{4}-(2 M+6 Q) .
\end{aligned}
$$



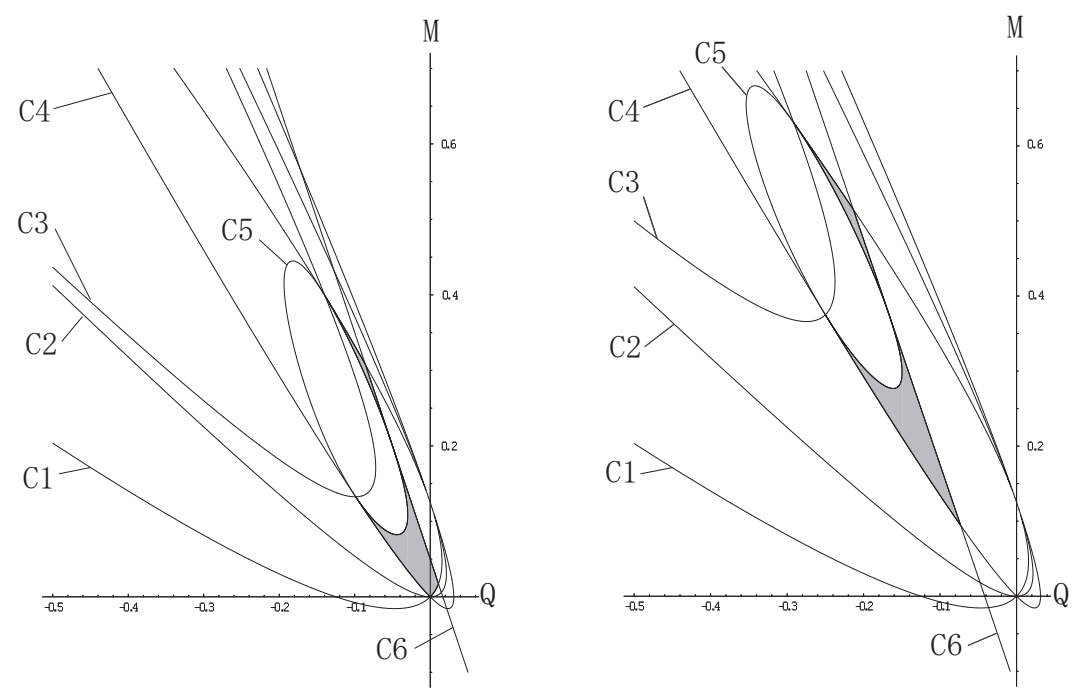

FIG. 2: The shaded regions in the left and right figures denote the parameter regions in the cases of $R_{\infty}^{2}=0.15$ and $R_{\infty}^{2}=0.50$, respectively.
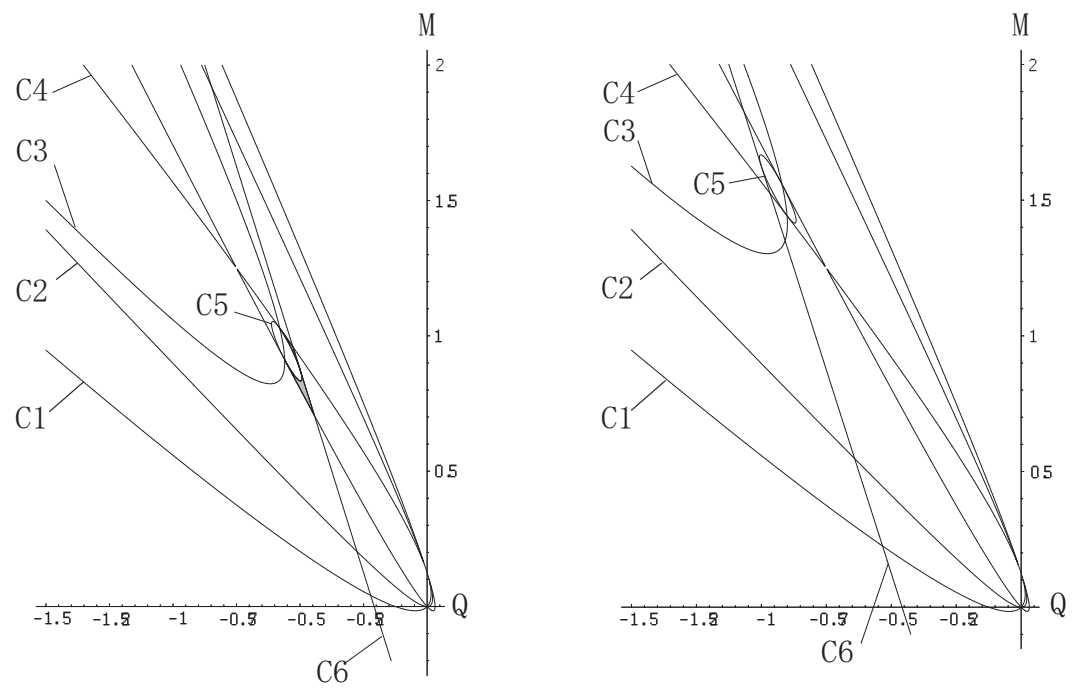

FIG. 3: The left and right figures correspond to the cases of $R_{\infty}^{2}=2.00$ and $R_{\infty}^{2}=3.00$, respectively. As $R_{\infty}$ increases, the curve $(C 6)$, which comes from the condition for the absence of CTCs, shifts below. So if $R_{\infty}$ takes sufficiently large values, then CTCs appear outside the outer horizon and the parameter region disappears. 


\section{B. Parameter region}

Here we derive the parameter region (30)-(34). The Killing horizons are located at the root $r>0$ of $V(r)=0$, i.e., the quadratic equations with respect to $r^{2}$ :

$$
r^{4}+\left[-2 m+16 j^{2}(m+q)(m+2 q)\right] r^{2}+q^{2}\left(1-8 j^{2}(m+3 q)\right)=0 .
$$

This equation has two different positive real roots within the range of $\left(0, r_{\infty}^{2}\right)$ if and only if the parameters in the solution obey the inequalities

$$
\begin{aligned}
& r_{\infty}^{2}>m-8 j^{2}(m+q)(m+2 q)>0, \\
& q^{2}\left(1-8 j^{2}(m+3 q)\right)>0, \\
& r_{\infty}^{4}+\left[-2 m+16 j^{2}(m+q)(m+2 q)\right] r_{\infty}^{2}+q^{2}\left(1-8 j^{2}(m+3 q)\right)>0, \\
& {\left[m-q-8 j^{2}(m+q)^{2}\right]\left[m+q-8 j^{2}(m+2 q)^{2}\right]>0 .}
\end{aligned}
$$

To avoid the existence of CTCs outside the horizons, we have to choose the parameters so that the two-dimensional part of the metric spanned by $(\psi, \phi)$ is positive-definite everywhere outside the outer horizon, i.e., $g_{\phi \phi}>0$ and $g_{\psi \psi}>0$. This is achieved whenever $g_{\psi \psi}>0$ since $g_{\phi \phi}=g_{\psi \psi} \cos ^{2} \theta+(1 / 4) r^{2} k(r) \sin ^{2} \theta$. For this, the following inequality needs to be satisfied everywhere in the region $\left(r_{+}^{2}, r_{\infty}^{2}\right)$,

$$
h(r)+\frac{r^{2}}{4}>0 \Longleftrightarrow u\left(r^{2}\right):=-4 j^{2} r^{2}+\left(1-4 j^{2}(2 m+6 q)\right)>0 .
$$

Since $u$ is a monotonically decreasing function of $r^{2}$, the necessary and sufficient condition for $u>0, r^{2} \in\left(r_{+}^{2}, r_{\infty}^{2}\right)$ is simply $u\left(r_{\infty}^{2}\right)>0$. Hence the necessary and sufficient condition for the absence of CTCs outside the horizons is given by eq. (34).

\section{Asymptotic structure and asymptotic charges}

In the coordinate system $(t, r, \theta, \phi, \psi)$, the limit $r \rightarrow r_{\infty}$ corresponds to spatial infinity. To investigate the asymptotic structure of the solution, as in the case of the rotating GPS monopole background, we introduce the new radial coordinate defined by

$$
\rho=\rho_{0} \frac{r^{2}}{r_{\infty}^{2}-r^{2}},
$$


which is similar to eq. (13) but the positive constant $\rho_{0}$ is now given by

$$
\rho_{0}^{2}=\frac{r_{\infty}^{2}}{4} V\left(r_{\infty}\right)
$$

Moreover, we introduce coordinates $(\bar{t}, \bar{\psi})$ so that the metric is in a rest frame at infinity

$$
d t=A d \bar{t}, \quad d \psi=d \bar{\psi}+B d \bar{t}
$$

where two constants $A$ and $B$ are chosen as

$$
\begin{aligned}
A & =\frac{\sqrt{1-4 j^{2}\left(2 m+6 q+r_{\infty}^{2}\right)} r_{\infty}^{2}}{\sqrt{\left(q^{2}-2 m r_{\infty}^{2}\right)\left(1-8 j^{2}(m+3 q)\right)+r_{\infty}^{4}+32 j^{2} q^{2} r_{\infty}^{2}}} \\
B & =\frac{4 j\left(3 q+r_{\infty}^{2}\right)}{\sqrt{\left.1-4 j^{2}\left(2 m+6 q+r_{\infty}^{2}\right)\right)} \sqrt{\left(q^{2}-2 m r_{\infty}^{2}\right)\left(1-8 j^{2}(m+3 q)\right)+r_{\infty}^{4}+32 j^{2} q^{2} r_{\infty}^{2}}}
\end{aligned}
$$

Then, for $\rho \rightarrow \infty$, the metric behaves as

$$
d s^{2} \simeq-d \bar{t}^{2}+d \rho^{2}+\rho^{2}\left(\sigma_{1}^{2}+\sigma_{2}^{2}\right)+L^{2} \sigma_{3}^{2}
$$

where the angular coordinate $\psi$ in $\sigma_{3}$ is replaced by $\bar{\psi}$ and $L$ is given by

$$
L^{2}=\frac{r_{\infty}^{2}}{4}\left[1-4 j^{2}\left(2 m+6 q+r_{\infty}^{2}\right)\right] .
$$

The constant $L$ is real under the condition, eq. (34), and describes the size of the extra dimension, as indicated from the asymptotic form of the metric, eq. (52).

The Komar mass and the Komar angular-momenta at spatial infinity are given, respectively, by

$$
\begin{aligned}
M_{K} & =-3 \pi \frac{\left(m r_{\infty}^{2}-q^{2}\right) k^{2}-j^{2} r_{\infty}^{2}\left(10 q^{2}+8 m r_{\infty}^{2}\right) k+128 j^{2} q^{2} r_{\infty}^{4}+2 j^{2} r_{\infty}^{6}}{4 \sqrt{\left(\left(q^{2}-2 m r_{\infty}^{2}\right) k^{2}-2 m r_{\infty}^{2}+r_{\infty}^{4}\right)\left(k-4 j^{2} r_{\infty}^{2}\right)}} \\
J_{\psi} & =\frac{\pi}{4} j r_{\infty}^{2}\left[3 q\left(1-8 j^{2}\left(m+r_{\infty}^{2}\right)-72 j^{2} q^{2}-4 j^{2} r_{\infty}^{4}\right)\right] \\
J_{\phi} & =0
\end{aligned}
$$

where $k:=1-8 j^{2}(m+3 q)$. Therefore the spacetime has the angular momentum only in the direction of the extra-dimension, i.e., along $\psi^{\mu}$, as is the case of the rotating GPS monopole. It is clearly seen from eq. (55) that the rotation with the angular momentum $J_{\psi}$-being proportional to $j r_{\infty}^{2}$-is a consequence of a combined effect of the background Gödel rotation and the squashing transformation. The global charge is expressed as

$$
\mathrm{Q}_{\mathbf{e}}=\frac{1}{4 \pi} \int\left(* F-\frac{2}{\sqrt{3}} A \wedge F\right)=-\frac{\pi \sqrt{3}}{2} q\left(1-8 j^{2}(m+q)\right) .
$$




\section{Near-Horizon Geometry and Regularity}

As mentioned above, the hypersurfaces at $r=r_{ \pm}$, where $V\left(r_{ \pm}\right)=0$, are Killing horizons. To confirm this, we introduce new coordinates $\left(v, \psi^{\prime}\right)$ defined by

$$
\begin{aligned}
& t=v+\int \frac{2 \sqrt{h(r)+r^{2} / 4} k(r)}{r V(r)} d r \\
& \psi=\psi^{\prime}+\int \frac{2 k(r) g(r)}{r V(r) \sqrt{h(r)+r^{2} / 4}} d r+\frac{g\left(r_{ \pm}\right)}{h\left(r_{ \pm}\right)+r_{ \pm}^{2} / 4} v .
\end{aligned}
$$

In a neighborhood of $r=r_{ \pm}$, the metric behaves as

$$
d s^{2} \simeq-\frac{r_{ \pm} k\left(r_{ \pm}\right)}{2 \sqrt{h\left(r_{ \pm}\right)+r_{ \pm}^{2} / 4}} d v d r+\left[\frac{r_{ \pm}^{2}}{4} k\left(r_{ \pm}\right)\left(\sigma_{1}^{2}+\sigma_{2}^{2}\right)+\left(h\left(r_{ \pm}\right)+\frac{r_{ \pm}^{2}}{4}\right) \sigma_{3}^{2}\right]+\mathcal{O}\left(\left(r-r_{ \pm}\right)\right)(6
$$

where the angular coordinate $\psi$ in $\sigma_{3}$ has been replaced with $\psi^{\prime}$. The Killing vector field $v^{\mu}=(\partial / \partial v)^{\mu}$ becomes null on $r=r_{ \pm}$and, furthermore, it is straightforward to see that $v^{\mu}$ is hypersurface-orthogonal at $r=r_{ \pm}$. Therefore the hypersurfaces $r=r_{ \pm}$are Killing horizons. Note also that in the coordinate system $\left(v, \phi, \psi^{\prime}, r, \theta\right)$, each component of the metric is analytic on and outside the black hole event horizon. Hence the space-time has no curvature singularity on and outside the black hole horizon.

\section{E. Shape of the horizons}

Now we investigate the shape of the horizons, especially, the ratio of $S^{2}$ base space to $S^{1}$ fiber. The horizon cross-section metric is

$$
\left.d s^{2}\right|_{r=\text { const. }}=\frac{r^{2}}{4} k(r)\left(\sigma_{1}^{2}+\sigma_{2}^{2}\right)+\left(h(r)+\frac{r^{2}}{4}\right) \sigma_{3}^{2} .
$$

The squashing of $\mathrm{S}^{3}$ is denoted by the ratio $k(r) /\left(h(r)+r^{2} / 4\right)$. In the case of $k\left(r_{ \pm}\right) /\left(h\left(r_{ \pm}\right)+\right.$ $r / 4)>1$, the surface is called oblate, since the radius of $S^{2}$ is larger than that of $\mathrm{S}^{1}$. In the case of $k\left(r_{ \pm}\right) /\left(h\left(r_{ \pm}\right)+r_{ \pm}^{2} / 4\right)<1$, it is called prolate, since the radius of $\mathrm{S}^{2}$ is smaller than that of $\mathrm{S}^{1}$. In the case of $k\left(r_{+}\right) / h\left(r_{+}\right)=1$, it is called a round $\mathrm{S}^{3}$. In the static case [29], the outer horizon is always oblate. In contrast, in the rotating case [33], the horizon admits a prolate shape in addition to the round $S^{3}$. In our solution, the outer horizon is always 
oblate, i.e.,

$$
\frac{r_{+}^{2} k\left(r_{+}\right)}{4 h\left(r_{+}\right)+r_{+}^{2}}>1,
$$

and the inner horizon is always prolate, i.e.,

$$
\frac{r_{-}^{2} k\left(r_{-}\right)}{4 h\left(r_{-}\right)+r_{-}^{2}}<1 .
$$

\section{SPECIAL CASES}
A. $\quad j \rightarrow 0$

In the limit $j \rightarrow 0$, the solution coincides with the static charged Ishihara-Matsuno solution to the five-dimensional Einstein-Maxwell equations. In particular, in the case of $r_{\infty} \rightarrow \infty$, i.e., $k(r) \rightarrow 1$, the solution becomes the five-dimensional Reissner-Nordström solution, which is asymptotically flat in the standard five-dimensional sense. In the case $m= \pm q$, it has a degenerate horizon and is supersymmetric because it is included in a class of solutions on Taub-NUT base space in [49], in which all purely bosonic supersymmetric solutions of minimal supergravity in five dimensions are classified. In fact, taking the limit of $m \rightarrow \pm q$ with introducing new coordinates $(\tilde{t}, \tilde{r})$ and the parameters $\left(\tilde{Q}, R_{\infty}\right)$ defined as

$$
\begin{aligned}
& r^{2}=\frac{4\left(R_{\infty}^{2} \tilde{r}+R_{\infty} \tilde{Q}\right)}{\tilde{r}+R_{\infty}}, \\
& t=\frac{R_{\infty}^{2}}{R_{\infty}^{2}-\tilde{Q}} \tilde{t} \\
& r_{\infty}^{2}=4 R_{\infty}^{2}, \\
& q= \pm 4 \tilde{Q}
\end{aligned}
$$

we obtain the following metric

$$
d s^{2}=-H^{-2} d \tilde{t}^{2}+H d s_{\mathrm{T}-\mathrm{NUT}}^{2}
$$

Here $d s_{\mathrm{T}-\mathrm{NUT}}^{2}$ is the metric on the Euclidean self-dual Taub-NUT space and is given, in terms of the Gibbons-Hawking coordinates, by

$$
d s_{\mathrm{T}-\mathrm{NUT}}^{2}=H_{k}\left(d \tilde{r}^{2}+\tilde{r}^{2} d \Omega_{S^{2}}^{2}\right)+R_{\infty}^{2} H_{k}^{-1} \sigma_{3}^{2}
$$


where $H$ and $H_{k}$ are harmonic functions on the three-dimensional Euclid space and are expressed in the present coordinate system as

$$
\begin{aligned}
& H=1+\frac{\tilde{Q}}{R_{\infty} \tilde{r}}, \\
& H_{k}=1+\frac{R_{\infty}}{\tilde{r}} .
\end{aligned}
$$

This metric, eq. (68), coincides with the metric of the supersymmetric static black hole solutions with a compactified extra dimension on the Euclidean self-dual Taub-NUT space in Ref. [34]. Moreover, in the case of $r_{\infty} \rightarrow \infty$, the metric can be written as

$$
d s^{2}=-\left(1 \pm \frac{q}{R^{2}}\right)^{-2} d t^{2}+\left(1 \pm \frac{q}{R^{2}}\right)\left[d R^{2}+R^{2} d \Omega_{S^{3}}^{2}\right],
$$

where $R^{2}=r^{2} \mp q$ and $d \Omega_{S^{3}}^{2}$ is the metric on the unit round three-sphere. This is the metric of the extremal Reissner-Nordstöm black hole solution which is supersymmetric solution on a four-dimensional Euclidean base space.

\section{B. Extremal case}

As shown previously, the Killing horizons are located at $r=r_{ \pm}$. In either the case $m-q-8 j^{2}(m+q)^{2}=0$ or the case $m+q-8 j^{2}(m+2 q)=0$, the two horizons degenerate, i.e., $r_{+}=r_{-}$. Note however that as far as the Gödel parameter $j$ is non-vanishing, the extremal limit of the present solutions are not supersymmetric in the sense of [49].

\section{Neutral cases}

For the Ishihara-Matsuno [29] and the rotating Ishihara-Matsuno solution [33], the parameter, $q$, is simply in proportion to the physical charge $Q_{e}$. However, for the present solution, this is not the case due to the Gödel parameter $j$, as seen in eq. (57). The physical global charge, $Q_{e}$, for the present solution vanishes in the case of either $q=0$ or $1-8 j^{2}(m+q)=0$.

For the former case $(q=0)$, the solution coincides with the squashed SchwarzschildGödel black hole solution [25], which is obtained via the squashing transformation for the 
Schwarzschild-Gödel black hole solution in Ref. [32]. In this case, the parameter region for the existence of a black hole horizon and non-existence of CTCs outside the horizon becomes

$$
\begin{aligned}
& m>0, \\
& 1-8 j^{2} m>0, \\
& -2 m+\frac{1}{4 j^{2}}>r_{\infty}^{2}>2 m\left(1-8 j^{2} m\right) .
\end{aligned}
$$

The horizon is located at $r=r_{\mathcal{H}}$ satisfying

$$
r_{\mathcal{H}}^{2}=2 m\left(1-8 j^{2} m\right)
$$

The shaded region in FIG 4 shows the region in which $(m, j)$ satisfy the inequalities (73)(75). Ergoregions are located in the regions such that $F\left(r^{2}\right)=r^{4} g_{\overline{t t}}$ is positive, where the function $F\left(r^{2}\right)$ is a cubic equation with respect to $r^{2}$, and is explicitly written as

$$
\begin{aligned}
F\left(r^{2}\right)= & -16 j^{4} r_{\infty}^{8} r^{6}-4 j^{2} r_{\infty}^{8}\left(1-8 j^{2}\left(m+r_{\infty}^{2}\right)\right) r^{4} \\
& -\left[r_{\infty}^{4}\left(1-4 j^{2}\left(2 m+r_{\infty}^{2}\right)\right)\right]^{2} r^{2}+2 m r_{\infty}^{8}\left[1-2 j\left(2 j\left(2 m+r_{\infty}^{2}\right)\right)\right]^{2}
\end{aligned}
$$

Note that $g_{\overline{t t}}\left(r=r_{\mathcal{H}}\right)>0$ and $g_{\overline{t t}}\left(r=r_{\infty}\right)<0$ always hold. Hence the boundary of the ergoregion is always located at $r$ such that $g_{\overline{t t}}=0$. Interestingly, when $F\left(\alpha^{2}\right)<0$ and $r_{\mathcal{H}}<\alpha$, there exist two ergoregions $r_{\mathcal{H}}<r<r_{1}$ and $r_{2}<r<r_{3}$ outside the black hole horizon, where $\alpha^{2}$ and $\beta^{2}(0<\alpha<\beta)$ are the roots of the quadratic equation with respect to $r^{2}, \partial_{r^{2}} F\left(r^{2}\right)=0$, and $r_{i}^{2}\left(i=1,2,3, r_{1}<r_{2}<r_{3}\right)$ are the roots of the cubic equation with respect to $r^{2}, F\left(r^{2}\right)=0$. The small dark region in FIG 5 denotes the set of the solutions which admit two ergoregions outside the horizon.

For the latter case (i.e., $\left.1-8 j^{2}(m+q)=0\right)$, FIG. 6 shows the line of $1-8(M+Q)=0$ in the $(Q, M)$-plane for the four values of $R_{\infty}^{2}=0.15,0.50,1.00,1.50$. In the case $R_{\infty}^{2}=$ $0.15,0.50,1.00$, the line, $1-8(M+Q)=0$, penetrates through the allowed parameter region, whereas in the case $R_{\infty}^{2}=1.50$, it does not. 


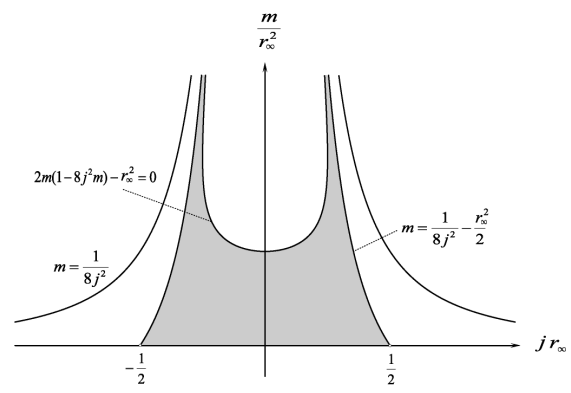

FIG. 4: Parameter region of the squashed Schwarzschild-Gödel black hole solution in the $\left(j r_{\infty}, m / r_{\infty}^{2}\right)$-plane.



FIG. 5: In the small dark parameter region in the above figure, there are two ergoregions. The below figure shows the close-up of the dark region in the above figure. 



FIG. 6: The bold lines show the lines where the global charge $Q_{e}$ vanishes for $(i) R_{\infty}^{2}=$ $0.15,($ ii $) R_{\infty}^{2}=0.50$, (iii) $R_{\infty}^{2}=1.00,($ iv $) R_{\infty}^{2}=1.50$. 


\section{SUMMARY AND CONCLUSIONS}

In this paper, we have presented a new class of stationary charged Kaluza-Klein black hole solutions, which are constructed from the Reissner-Nordstöm-Gödel black hole solution in five-dimensional Einstein-Maxwell-Chern-Simons theories, via the squashing transformation. Our solutions can be viewed as a charged black hole in the rotating GPS monopole background and be seen to be rotating by the effect of the rotation of the background GPS monopole. (Note that the rotation of the GPS background itself is caused by the existence of the non-homogeneous electromagnetic field.) Like known charged black hole solutions, our black hole spacetime has two horizons, the outer and inner horizons. Although the crosssection geometry of the outer horizon is of squashed $\mathrm{S}^{3}$ (hence essentially a five-dimensional black hole), at large distances the spacetime behaves effectively as a four-dimensional spacetime (provided that the scale of extra-dimension, $L$ given by eq. (53), is sufficiently small compared to other physically relevant scales at large distances).

Some further remarks are in order. Our solutions are specified by four parameters, $\left(m, q, r_{\infty}, j\right)$. When the Gödel parameter, $j$, vanishes, the solution becomes the IshiharaMatsuno black hole solution, which is a static charged Kaluza-Klein black hole solution in the five-dimensional Einstein-Maxwell theory, in which the parameters, $m$ and $q$, are directly related to the globally defined mass and the electric charge, respectively, and $r_{\infty}$ is proportional to the size of the extra-dimension. When both the $m$ and $q$ are zero, our solution reduces to the rotating GPS monopole solution.

It should be noted that the parameter $r_{\infty}$ has an upper bound which comes from the condition for the absence of closed timelike curves. This is in contrast to the fact that neither the squashed Kerr-Gödel black hole solution [25] nor the rotating Ishihara-Matsuno solution [33] has CTCs (outside the black hole horizon) for any $r_{\infty}<\infty$. [52]

In the Ishihara-Matsuno solution [29], the $m= \pm q$ case corresponds to a supersymmetric solution, i.e., the static black hole on Taub-NUT space [49]. However, in the rotating Ishihara-Matsuno solution [33], the two cases, $m=-q$ and $m=q$, describe different solutions due to the existence of a Chern-Simons term. Only the case of $m=-q$ corresponds to a supersymmetric Kaluza-Klein black hole solution of Gaiotto et al [34]. In our solution, 
neither the case $m=-q$ nor the case $m=q$, is supersymmetric and the two horizons do not degenerate. For either $m-q=8 j^{2}(m+q)^{2}$ or $m+q=8 j^{2}(m+2 q)$, the outer and inner horizons degenerate, but in either case our solution is non-supersymmetric.

Finally we would like to make two comments on the squashing transformation. First, we recall that our starting-point geometry (either the Reissner-Nordstöm-Gödel black hole solution or the five-dimensional Gödel universe) suffers from causality violation, but nevertheless our resulting solutions do not. In view of this, we can anticipate that the squashing transformation may be used to generate much larger variety of physically interesting solutions, starting not only from physically sensible geometries (e.g., those of asymptotically flat, with no CTCs, etc) but even from various causally or metrically pathological geometries such as spacetimes with CTCs and/or naked singularities. Second, as far as the present authors know, the squashing transformation has so far been applied only to cohomogeneity-one black hole solutions such as five-dimensional Myers-Perry black hole solutions with two equal angular momenta and the Cvetic-Lu-Pop's charged black hole solutions with two equal angular momenta. It would be interesting to consider a generalization of the squashing technique so that it would apply to cohomogeneity-two spacetimes such as black ring [2], black holes with two rotations [1], black lens [50, 51], or even wider class of spacetimes.

\section{Acknowledgements}

AI wishes to thank the Perimeter Institute for Theoretical Physics for its hospitality during the time some of this research was carried out. This research was supported in part by the Japan Society for the Promotion of Science and in part by Perimeter Institute for Theoretical Physics.

\section{APPENDIX A: SUPERSYMMETRIC ROTATING GPS MONOPOLE}

Here we show that our background rotating GPS monopole solution is supersymmetric, using the results of Ref. [49]. We first recall that according to [49], all supersymmetric solutions of the five-dimensional minimal supergravity have a non-spacelike Killing vector 
field, and when the Killing vector field $\partial / \partial t$ is timelike, the metric and the gauge potential are given, respectively, by

$$
d s^{2}=-H^{-2}(d t+\boldsymbol{\omega})^{2}+H d s_{\mathcal{B}}^{2}, \boldsymbol{A}=\frac{\sqrt{3}}{2}\left[H^{-1}(d t+\boldsymbol{\omega})-\boldsymbol{\beta}\right]
$$

where $d s_{\mathcal{B}}^{2}$ is a metric of a hyper-Kähler space $\mathcal{B}$. The scalar function $H$, one-forms $\boldsymbol{\omega}$ and $\boldsymbol{\beta}$ on $\mathcal{B}$ are given by

$$
\Delta H=\frac{4}{9}\left(G^{+}\right)^{2}, \quad d G^{+}=0, \quad d \boldsymbol{\beta}=\frac{2}{3} G^{+} .
$$

Here, $\triangle$ is the Laplacian on $\mathcal{B}$ and the two-form $G^{+}$is the self-dual part of the one-form $H^{-1} \boldsymbol{\omega}$, given by

$$
G^{+}:=\frac{1}{2} H^{-1}(d \omega+* d \omega)
$$

where $\left(G^{+}\right)^{2}:=\frac{1}{2} G_{m n} G^{m n}$ and $*$ is the Hodge dual operator on $\mathcal{B}$. Since $\partial / \partial t$ is a Killing vector field associated with time translation, all components are independent of the time coordinate $t$.

We now show that the rotating GPS monopole solution takes the form (A1)-(A3). Comparing eq. (14) and eq. (A1), we can read off the correspondence of these two metrics as follows

$$
\begin{gathered}
H=1, \quad \boldsymbol{\omega}=4 j V \sigma_{3}, \\
d s_{\mathcal{B}}^{2}=V^{-1}\left(d r^{2}+r^{2} d \Omega_{S^{2}}^{2}\right)+V \rho_{0}^{2}(d \psi+\cos \theta d \phi)^{2},
\end{gathered}
$$

with

$$
V^{-1}=1+\frac{\rho_{0}}{\rho}
$$

where $d s_{\mathcal{B}}^{2}$ is the metric of the Euclidean self-dual Taub-NUT space and $V^{-1}$ is a harmonic function on the three-dimensional Euclid space $\mathbb{E}^{3}$. Note that $d \sigma_{3}=-\sigma_{1} \wedge \sigma_{2}$. So the exterior derivative of the one-form $\boldsymbol{\omega}$ can be written as

$$
d \boldsymbol{\omega}=4 j\left(\frac{\rho_{0}}{\rho^{2}} V^{2} d \rho \wedge \sigma_{3}-V \sigma_{1} \wedge \sigma_{2}\right) .
$$


Then the dual of the one-form $\omega$ is obtained as

$$
* d \boldsymbol{\omega}=-4 j\left(\frac{\rho_{0}}{\rho^{2}} V^{2} d \rho \wedge \sigma_{3}-V \sigma_{1} \wedge \sigma_{2}\right)
$$

As a result, we find

$$
G^{+}=0
$$

The second equation eq. (A2) is automatically satisfied. The first equation of eq. (A2) becomes

$$
\Delta_{T-N U T} H=0
$$

and obviously, $H=1$ solves this equation. The third equation of eq. (A2) yields $d \boldsymbol{\beta}=0$. Hence the one-form $\boldsymbol{\beta}$ takes a closed form at least locally, i.e., there exists some function $\gamma$ such that $\boldsymbol{\beta}=d \gamma$. We can set the function $\gamma$ to zero by the gauge transformation. Thus the rotating GPS monopole solution (14)-(15) is supersymmetric.

[1] R.C. Myers and M.J. Perry, Annals Phys. 172, 304 (1986).

[2] R. Emparan and H.S. Reall, Phys. Rev. Lett. 88, 101101 (2002).

[3] T. Mishima and H. Iguchi, Phys. Rev. D 73, 044030 (2006).

[4] A.A. Pomeransky and R.A. Sen'kov, hep-th/0612005.

[5] P. Figueras, JHEP 0507, 039 (2005).

[6] H. Iguchi and T. Mishima, Phys. Rev. D 75, 064018 (2007).

[7] H. Elvang and P. Figueras, JHEP 0705, 050 (2007).

[8] J.C. Breckenridge, R.C. Myers, A.W. Peet and C. Vafa, Phys. Lett. B 391, 93 (1997).

[9] H. Elvang, R. Emparan, D. Mateos, H. S. Reall, Phys. Rev. Lett. 93, 211302 (2004).

[10] H. Elvang, R. Emparan, D. Mateos, H. S. Reall, Phys. Rev. D 71, 024033 (2005).

[11] J. P. Gauntlett and J. B. Gutowski, Phys. Rev. D 71, 025013 (2005).

[12] J. P. Gauntlett and J. B. Gutowski, Phys. Rev. D 71, 045002 (2005).

[13] M. Cvetic and D. Youm, Nucl. Phys. B 476, 118 (1996); M. Cvetic, H. Lu and C.N. Pope, Phys. Lett B 598, 273 (2004). 
[14] M. I. Cai and G. J. Galloway, Class. Quant. Grav. 18, 2707 (2001).

[15] C. Helfgott, Y. Oz and Y. Yanay, JHEP 0602, 025 (2006).

[16] G. J. Galloway and R. Schoen, Commun. Math. Phys. 266, 571, (2006).

[17] Y. Morisawa and D. Ida, Phys. Rev. D 69, 124005 (2004).

[18] Y. Morisawa, S. Tomizawa and Y. Yasui, Phys. Rev. D 77, 064019 (2008).

[19] S. Hollands, A. Ishibashi and R. M. Wald, Commun. Math. Phys. 271, 699 (2007).

[20] N. Arkani-Hamed, S. Dimopoulos and G. Dvali, Phys. Lett. B 429, 263 (1998).

[21] L. Randall and R. Sundrum, Phys. Rev. Lett. 83, 3370 (1999).

[22] L. Randall and R. Sundrum, Phys. Rev. Lett. 83, 4690 (1999).

[23] C. A. R. Herdeiro, Class. Quant. Grav. 20, 4891, (2003).

[24] D.J. Gross and M. J. Perry, Nucl. Phys. B 226, 29 (1983);

R.D. Sorkin, Phys. Rev. Lett. 51, 87 (1983).

[25] S. Tomizawa, H. Ishihara, K. Matsuno and T. Nakagawa, arXiv:0803.3873 [hep-th].

[26] P. Dobiasch and D. Maison, Gen. Rel. Grav. 14, 231 (1982).

[27] G.W. Gibbons and D.L. Wiltshire, Ann. Phys. 167, 201 (1986).

[28] D. Rasheed, Nucl. Phys. B, 454, 379, (1995).

[29] H. Ishihara and K. Matsuno, Prog. Theor. Phys. 116, 417 (2006).

[30] H. Ishihara, M. Kimura, K. Matsuno and S. Tomizawa, Class. Quant. Grav. 23, 6919 (2006).

[31] T. Wang, Nucl. Phys. B, 756, 86 (2006).

[32] E. Gimon and A. Hashimoto, Phys. Rev. Lett. 91, 021601 (2003).

[33] T. Nakagawa, H. Ishihara, K. Matsuno and S. Tomizawa, Phys. Rev. D 77, 044040 (2008).

[34] D. Gaiotto, A. Strominger and X. Yin Yin, JHEP 02, 024 (2006).

[35] H. Elvang, R. Emparan, D. Mateos and H. S. Reall, JHEP 0508, 042 (2005).

[36] R. C. Myers, Phys. Rev. D, 35, 455 (1987).

[37] H. Iguchi, T. Mishima and S. Tomizawa, Phys. Rev. D 76, 124019 (2007).

[38] S. Tomizawa, H. Iguchi and T. Mishima, e-Print: hep-th/0702207.

[39] I. Bena, P. Kraus and N.P. Warner, Phys. Rev. D 72, 084019 (2005).

[40] T. Harmark and N. A. Obers, e-Print: arXiv: hep-th/0503020.

[41] D. Kastor, S. Ray and J. Traschen, Class. Quant. Grav. 25, 125004 (2008). 
[42] H. Ishihara, M. Kimura, K. Matsuno and S. Tomizawa, Phys. Rev. D 74, 047501 (2006).

[43] H. Ishihara, M. Kimura, S. Tomizawa, Class. Quant. Grav. 23, L89 (2006).

[44] K.Matsuno, H. Ishihara, M. Kimura and S. Tomizawa, Phys. Rev. D 76, 104037 (2007).

[45] S. Tomizawa, H. Ishihara, M. Kimura and K. Matsuno, Class. Quant. Grav. 24, 5609 (2007).

[46] S. Tomizawa, Class. Quant. Grav. 25, 145014 (2008).

[47] D. Ida, H. Ishihara, M. Kimura, K. Matsuno, Y. Morisawa and S. Tomizawa, Class. Quant. Grav. 24, 3141 (2007).

[48] C.-M. Yoo, H. Ishihara, M. Kimura, K. Matsuno and S. Tomizawa, Class. Quant. Grav. 25, 095017 (2008).

[49] J.P. Gauntlett, J.B. Gutowski, C.M. Hull, S. Pakis and H.S. Reall, Class. Quant. Grav. 20, 4587 (2003).

[50] H. Lu, Jianwei Mei and C.N. Pope, e-Print: arXiv:0804.1152 [hep-th].

[51] H. Lu, Jianwei Mei and C.N. Pope, e-Print: arXiv:0806.2204 [hep-th].

[52] Note that the squashed Kerr-Newman Gödel black hole solution -which has 5 parameters $m, q, j, r_{\infty}$ and the angular-momentum parameter $a$ - has recently been constructed in [25]. However, it would not appear to be straightforward to specify possible parameter region for which CTCs cease to exist outside the black hole horizon of this solution. 